\begin{tabular}{c}
\hline \hline Current Topics \\
Advances in Immuno- \\
therapy Strategies \\
\hline \hline Juntendo Medical Journal \\
$2015.61(6), 580-587$
\end{tabular}

\title{
The Current State of Immune Therapy in Multiple Sclerosis in Japan and Future Prospects
}

\author{
KAZUMASA YOKOYAMA*, NOBUTAKA HATTORI* \\ *Department of Neurology, Juntendo University Faculty of Medicine, Tokyo, Japan
}

\begin{abstract}
Among neuroimmunological disorders, the most common disease is multiple sclerosis (MS). MS is an intractable disease particularly affecting young adults and is increasing all over the world, including Japan. The main goal of treatment is to decrease the frequency of relapses, manage symptoms, improve function, and delay disease progression for good quality of life. To prevent relapses, interferon beta $1 \mathrm{~b}$, an injectable disease-modifying therapy (DMT), has been used; however, it has been only available in Japan since 2000, about seven years behind world usage, because of the drug lag caused by healthcare system barriers. At present, second-line DMTs, such as fingolimod and natalizumab, are available; both are more effective compared to first-line injectable interferons and glatiramer acetate (approved on September 28, 2015). However, it is still difficult to block the neurodegenerative process of axonal damage, and that is why there is no cure for MS. In this article, we summarize current immunotherapy in Japan and discuss the pros and cons of the newer DMTs, which might modulate disease course. We also discuss the future prospects of DMTs. Even if DMT treatment is initiated immediately after the completion of diagnosis, patients need to remain on therapy for a long period of time. Adhering patients to treatment is another important aspect. In this regard, a treatment algorithm for escalation therapy or early aggressive induction therapy is not a final decision, and we need to reconsider other better options with low risk of drug adverse effect, and exclude pregnancy-related risks in females.
\end{abstract}

Key words: multiple sclerosis, disease modifying therapy, window of therapeutic opportunity, PML, NEDA, adherence

\section{Introduction}

Currently, in our department of Neurology, we treated neuroimmunological disorders including, multiple sclerosis (MS), neuromyelitis optica (NMO), acute disseminated encephalomyelitis (ADEM), myasthenia gravis (MG), Guillain-Barre syndrome, chronic inflammatory demyelinating polyneuropathy (CIDP), neurosarcoidosis, neuro-Behcet, neuroSweet disease, Hashimoto encephalopathy, non herpetic autoimmune limbic encephalitis, Isaccs' disease and so on. We have started the new program 'Treatment and Research in Multiple Sclerosis and Neuro-intractable Disease UNIT (TRI-MS/NID)' in our department from October of 2014. The space is limited in this artcle, so we would like to concentrate onto current and future prospect of MS immunotherapy.

\section{What is MS?}

MS is thought to be as an autoimmune central nervous disorders affecting oligodendrocyte from acute inflammation and then chronic demyelination occurred. The types of MS are divided into 3 characteristic, one is relapsing remitting MS, patients have distinct attacks of symptoms which then recovered either partially or completely in especially earlier stage. After living with MS about 10-15 years, patients move on to a secondary progressive MS. Axonal loss due to inflammation is less clinically silent during the relapsing-remitting phase of the disease, and once a threshold for axonal transection is exceeded by undetermined patho-

Corresponding author: Kazumasa Yokoyama

Department of Neurology, Juntendo University Faculty of Medicine

2-1-1 Hongo, Bunkyo-ku,Tokyo 113-8421, Japan

TEL: +81-3-3813-3111 E-mail: kazumasa@juntendo.ac.jp

[Received Sep. 28, 2015] 
physiology, MS patients enter an irreversible progressive phase. Primary progressive MS showed no well-defined attacks of symptoms but just the symptoms march and showed no response to any treatment so far in the long run.

\section{When to start DMTs?}

It is widely accepted in the rheumatology community that a 'therapeutic window of opportunity' exists for patients with rheumatoid arthritis (RA), that the disease is much more amenable to treatment within this window, and that treatment within the window can reset the disease's long-term trajectory in a way that treatment outside of it cannot $^{1)}$.

Likewise there is an idea that MS clinicians use DMTs for earlier stage of the disease without any state of progressive course (less degenerative change) that might be an efficacious window in the long term and should be insisted because rheumatologist can replace patients' joint for artificial one but neurologist could not change patient's brain.

In this context, the term "clinically isolated syndrome" (CIS) was the target of MS DMTs in North America and European countries. This definition was used to describe a first episode of neurologic symptoms that lasts at least 24 hours and was caused by inflammation and demyelination in one or more sites in the central nervous system (CNS). CIS developed clinically definite MS (CDMS fulfill the requirement of $2005 \mathrm{McD}$ onald's criteria) overtime in a percentage of patients ranging from $20 \%$ to $80 \%$, depending on the presence of certain baseline features ${ }^{2)}$.

Current evidence suggests that DMTs should be started at this stage since it is likely to have an important impact on the evolution of the disease ${ }^{3)}$ 4).

Unfortunately, in Japan we could not use DMTs onto CIS because CIS is not defined as MS and Japanese healthcare system would not support the medical expenses as for the intractable disease. By now, MS diagnostic criteria was revised in 2010 and definition of CIS is not the same, also there is no clinical trial of natalizumab and fingolimod in patients on CIS even in the western countries, therefore we could not understand the real world setting of efficacy and long term risk of both medicine.

\section{What strategy and how we evaluate disease activity?}

The treatment algorithms of MS in North America as well as European countries cannot be adopted formally in Japan. However we have yielded important data to select several treatment paradigms from different clinical trials conducted overseas. One is earlier induction therapy and the other is escalation therapy strategy, both are for the purpose of fulfill the treatment goal of disease free status as no evidence of disease activity (NEDA). NEDA was defined firstly by AFFIRM study of natalizumab phase 3 clinical trial ${ }^{5}$.

NEDA represents by the clinical disease-free status (no activity on clinical measures that is no relapses and no sustained disability progression), was combined with radiologic disease-free status (that is no gadolinium-enhancing lesions and no new enlarging T2-hyperintense lesions on brain MRI), and treatment goal and outcome measure of relatively early in the course of the disease.

The induction paradigm involves maximizing therapeutic benefit while tolerating increased therapeutic risk, based on the assumption that the risk of MS outweighs the risk of the treatment. The escalation paradigm involves minimizing therapeutic risk while maximizing therapeutic benefit, based on the assumption that one can decrease the risk of MS sufficiently while exposing the patient to less therapeutic risk ${ }^{6}$.

Some patient might achieve NEDA, however we could not predict the response of each individual in a long term. Because mode of mechanisms, efficacies, and safety of DMTs vary. Rotstein et al reported that CIS or MS patients recent onset $(<5$ year) and established ( $>5$ year) were enrolled in a seven-year longitudinal cohort of 215 patients with MS, $46 \%$ of patients on DMT reached NEDA at one year, but only $7.9 \%$ of the patients maintained NEDA for seven years even on DMTs. NEDA at 2 years had a positive predictive value of $78.3 \%$ for no progression (Expanded Disability Status Scale score change less than 0.5 ) at 7 years ${ }^{7)}$.

These results did not support the concept of therapeutic windows on induction therapy because of the recent onset and established RRMS, DMTs did not show any difference of NEDA ${ }^{7}$. Therefore we realized that DMTs delayed long-term disability 
in MS patients treated either in the early or, to a lesser extent, in the later phase of the disease. Thus, the window of therapeutic opportunity is relatively extended, assuming that early is better than late treatment, but late is better than never ${ }^{8}$.

\section{Is early aggressive induction therapy by second line DMTs a final goal?}

Aggressive induction therapy such as using fingolimod or natalizumab as a first line treatment is not universally accepted due to economical and lack of information about the long term adverse effects. Probably, the timing of the prognostic window of opportunity cannot be solved until the double endpoint of duration and age is consistently used, MRI and neurochemical parameters are taken into consideration and therapy is included as an active factor in the multivariate analyses ${ }^{9)}$.

\section{The current status of the MS DMTs in Japan}

As of today, there is no cure for MS but several DMTs were introduced from 1980's.

Currently, FDA approved 11 DMTs, the first disease DMT approved by the Food and Drug Administration (FDA) was interferon beta $1 \mathrm{~b}$ (Betaferon ${ }^{\circledR}$ ) in 1993 and clinical trial took place in 1996 and approved in 2000 in Japan. IFN-beta 1b (Avonex ${ }^{\circledR}$ ) followed in 2006, fingolimod (Gylenya ${ }^{\circledR}$, Imusera ${ }^{\circledR}$ ) in 2011, and natalizumab $\left(\right.$ Tysabri ${ }^{\circledR}$ ) in 2014. Another first line injectable glatiramer acetate (Copaxon ${ }^{\circledR}$ ) was approved on the $28^{\text {th }}$ of September 2015, 19 years behind. Phase III clinical trial of Demethyl fumarate $\left(\right.$ Tecifidera ${ }^{\circledR}$ ) are going on in Japan and wait for 2-3 years for approval. When we initiate the DMTs, we need to also consider adherence over a long term in order to reach acceptable outcomes. As Hansen et al reported, only $30-40 \%$ of patients were adherent to DMT treatment after two years ${ }^{10)}$.

It is influenced by frequency and route of administration, recent trend of per-oral medicine will show explosive increase of the usage.

\section{DMTs into detail}

\section{1) IFNs}

First line DMT such as interferons (beta 1 a Beta- feron ${ }^{\circledR}$ and b Avonex ${ }^{\circledR}$ in Japan) reduce relapse rate by approximately $30 \%$ and frequently associated with suboptimal response in terms of efficacy and several side effects such as injection-site reaction and flu-like symptoms, and liver dysfunction leading to poor patient adherence. The Rio score (RS) is a recently developed scoring system that consists of a combination of clinical and MRI parameters to predict suboptimal responders ${ }^{11)}$.

The Modified Rio score (MRS) is a simplified version of RS, which excludes expanded disability status scale (EDSS) progression and modified items of relapses and MRI lesions ${ }^{12}$.

These scores were estimated after 1 year of IFN beta therapy with the aim of identifying patients that will have ongoing disease activity and become suboptimal responders in the ensuing 2 years ${ }^{13)}$.

The expression of Sema4A is increased on DCs in MS patients and shed from these cells in a metalloproteinase-dependent manner. DC-derived Sema4A is not only critical for Th1 but also for Th17 cell differentiation, and MS patients with high Sema4A levels exhibit Th17 skewing. Furthermore, patients with high Sema4A levels have more severe disabilities and are unresponsive to IFN- $\beta$ treatment ${ }^{14)}$.

In Japan, we have a free support system for measuring Sema4A to predict the responsiveness of IFNs at Osaka University. When patients showed breakthrough for interferon we also have a free support system measuring neutralizing antibody for IFNs at Tohoku Univesity. Because neurralizing antibodies are associated with a reduction of IFN beta efficacy during the treatment ${ }^{15}$.

In terms of adherence, we are hoping to have a Pegylated form of subcutaneous interferon beta-1a approved by the FDA in 2014 with a longer half-life permits reduced injection frequency (every 2-4 weeks) ${ }^{16)}$.

\section{2) Glatiramer acetate}

This unique injectable (Copaxone ${ }^{\circledR}$ ) is produced by random polymer composed of four aminoacids with glutamic acid, lysine, alanine, and tyrosine, but the mechanism of action is unknown. The similarity of immunogenic epitope of myelin basic protein, $\mathrm{T}$ helper 2 shift might be one of the mechanisms. Daily subcutaneous injection is required, therefore $30 \%$ of the user showed injection site reaction and flu-like symptoms in about $10 \%$ of users. In occasion, 
lipoatrophy the local destruction of fat tissue may occur. Compare to IFNs, depression is less and efficacy in RRMS was $29 \%$ and used in CIS and efficacy is about $45 \%$ deduction of the transition to the clinically defined MS. FDA approved this medicine as category B during pregnancy (http://www. drugs.com/pro/copaxone.html).

Therefore many young female individuals wish to use this injectable. If we compare the two injectables it is reported that the main conclusion is that the two therapies do not seem to differ in terms of clinical efficacy and safety, although IFNs were found to limit the increase of MRI lesion burden more than GA. These data support clinicians in the use of these therapies based on their similarities in safety and efficacy in the prevention of disease activity, although the different effects on some MRI measures and the different levels of tolerability might have a role in therapeutic choice at the individual level. The assessment of patient-related outcomes is warranted to take into account the needs of patients in the treatment choice ${ }^{17}$.

\section{3) Fingolimod}

Fingolimod is the first oral drug approved FDA in 2010 originally produced in Japanese pharmaceutical company now in Mitsubishi Tanabe Pharma but licensed to Novartis for clinical trial worldwide. This is a first oral treatment for MS and used as first line in the United States and secondline therapy in Europe. In Japan, recommendation is for escalation therapy after treatment option for IFN non-responders. Fortunately, in Japan, we could use it for anyone who do not like injectables. Fingolimod are derived from myriocin, a metabolite of the ascomycete fungus Isaria sinclairii, and act like an endogenous lysophospholipid sphingosine-1 phosphate (SIP), first acting as a full S1PR agonist and thereafter functioning as an S1PR antagonist after down-regulation of S1PR subtype 1 on lymphocytes perturb the egress of lymphocytes from lymphoid tissue. At the same time various S1P receptors expressed in the CNS and fingolimod are lipophilic and easily cross BBB and modulate neurogenesis, neural function and migration and exerting neuroprotective or repair effects. Effectiveness is superior to IFN betala in A 12-month, double-dummy phase III study involving patients with RRMS but no significant differences were evident in terms of disability progression (TRANSFORMS: TRial Assessing INjectable INterferon vs. FTY720 Oral in RRMS) ${ }^{18)}$.

Fingolimod has been associated with transient dose-dependent bradycardia, atrioventricular conduction block, hypertension, macular edema, elevated liver enzyme levels, lymphocytopenia, and skin cancers in clinical trials in TRANSFORMS and FREEDOMS and two fatal infections occurred by varicella zoster and herpes simplex encephalitis ${ }^{19)}$.

The incidence of death including infection, suicide, lymphoma and cancer by fingolimod in Japan is very high. About one out of 400 treated patients died and the reason of this high mortality rate of fingolimod in Japan is still under investigation and pharmaceutical company should collect full data with transparency. Recently, it is reported that fingolimod treatment without prior treatment of natalizumab showed PML at least 3 cases in Gemany, Spain and United States. Right now the estimate incidence of PML is about 1.25/100000. That is less than the incidence of PML of natalizumab (564/142704) and even though stop treatment of natalizumab resulted in the death of 131 patients.

Fingolimod clinical trial in patients with primary progressive multiple sclerosis (INFORMS) was failed, which is a double-blind, randomized, multicenter, placebo-controlled parallel group study. Nine-hundred and seventy (970) people aged 25-69 years with PPMS were enrolled in INFORMS from 148 sites, across 18 countries. Patients were treated for at least three years. EXPAND study is to evaluate the safety and efficacy. Japan was not involved but Siponimod trial (BAF312 S1P1 selective antagonist) versus placebo in a variable treatment duration in patients with SPMS is still under investigation including Japan.

\section{4) Natalizumab}

Natalizumab is a humanized monoclonal antibody that selectively targets the alpha 4 subunit of the cell adhesion molecule "very late antigen4" expressed on the surface of lymphocytes and monocytes. It prevents the interaction between the very late antigen 4 integrin and its ligand vascular cell adhesion molecule-1 on brain vascular endothelium. This interaction is necessary for the transmigration of immune cells across the BBB; thus, circulating lymphocytes could not enter the CNS and trigger 
acute MS lesions ${ }^{20)}$.

FDA approved on 2004 but the first three patients with natalizumab-associated PML were described in 2005 they raised the level of awareness of the potential risks of PML with other therapeutic agents ${ }^{21)}$. An interruption to normal immune surveillance by natalizumab results in the escape of $\mathrm{JCV}$ to the CNS from sequestration in peripheral organs or primary CNS reactivation.

Risk factors for PML include evidence of prior JCV exposure, duration of natalizumab therapy, and prior use of immunosuppressants such as mitoxantrone, azathioprine, or methotrexate but not the immunomodulators interferon beta and GA or routine use of bolus corticosteroids for clinical relapses ${ }^{22)}$.

Owing to PML risk, natalizumab is generally reserved for use in patients with "breakthrough" disease activity on one or more of the first-line DMTs. Some MS specialists also use it as a first-line therapy for early aggressive MS for 1 to 2 years, to be followed by transition to another agent once disease activity appears controlled, especially in JCV seropositive individuals.

Patients treated fingolimod in other country after natalizumab cessation showed PML ${ }^{23)}$. Therefore we need take very care for detecting PML when patients treated with second line DMTs.

At present, among Japanese we have about $70 \%$ of JC virus antibody positivity measured by stratify method and so far we have no patients of PML.

The natalizumab molecule contains murine sequences that increase immunogenicity, resulting in infusion reactions and neutralizing antibodies ${ }^{24)}$. In practice, patients with persistent anti-natalizumab neutralizing antibody titers should discontinue therapy because of loss of efficacy and increased risk of hypersensitivity reactions, which may be fatal $^{25)}$.

Also there is issue of inflammatory activity of MS may return 3 to 6 months after the discontinuation of natalizumab ${ }^{26)}$, and switch therapy to fingolimod is less effective and $48 \%$ relapse, compare to finogolimod to natalizumab $(23 \%)^{27)}$.

In terms of PML, patients asymptomatic at diagnosis had better survival and less functional disability than those who were symptomatic at diagnosis. Immune reconstitution inflammatory syndrome (IRIS) was subsequently reported in 20 asymptomatic PML patients (66.7\%) and in 248 symptomatic PML patients (72.5\%). PML-IRIS was defined as worsening of clinical symptoms and lesion progression including signs of inflammation and mass effect on MRI, as determined by the reporting physician ${ }^{28)}$.

It is due to partly less expansion of the lesion and also less suffered from immune reconstitution inflammatory syndrome (IRIS) might be a better clinical outcome. Finally, disappointing result of the Phase 3 ASCEND (A Study to Characterize the Efficacy of Natalizumab on Disability in SPMS) study investigating natalizumab in the treatment of secondary progressive multiple sclerosis (SPMS) did not achieve its primary and secondary endpoints. The primary endpoint of the study was the percentage of patients with confirmed progression of disability on one or more components of ASCEND' s composite endpoint: the Expanded Disability Status Scale (EDSS), Timed 25-Foot Walk (T25FW) and the 9-Hole Peg Test (9HPT) where progression was confirmed at a second visit at least 6 months later and at week 96 .

\section{5) Dimethyl fumarate}

BG12 (Tecfidera ${ }^{\circledR}$ ) is a second-generation fumaric acid compound that contains only dimethylester, dimethylfumarate in oral formulation in the form of enteric-coated tablets, to minimize gastrointestinal ulcer formation ${ }^{29)}$.

Various mechanisms have been proposed to explain the effects of dimethyl fumarate in MS patients. The anti-inflammatory effects of the drug have been linked to ultimate reductions in lymphocyte counts and disruption of cell migration. Dimethyl fumarate and its primary metabolite, monomethyl fumarate, activate the nuclear erythroid-2-related factor 2 transcriptional pathway that controls expression of the gene encoding the phase- 2 detoxifying enzyme, which plays a crucial role in the oxidative stress response and immune homeostasis.

It was approved as first-line therapy for MS in 2013 in United States and European countries but still under phase 3 clinical trial in Japan, and will be approved for couple of years.

BG 12 is oral medicine approved next to fingolimod and believed safer from the long history of using fumaric acid esters (FAE) derived from 
the unsaturated dicarbonic acid fumaric acid in treatment of psoriasis in Germany, but also showed 3 PML. One had been part of a clinical trial and was taking the drug for $4-1 / 2$ years. The patient, who died of pneumonia, had been suffering severe lymphopenia, a low white blood cell condition, for more than three years. Periodic monitoring of absolute lymphocyte counts to identify patients at increased risk for severe, prolonged lymphocytopenia less than 500 cells $/ \mu l$ and consideration of treatment interruption in these patients may mitigate the risk of $\mathrm{PML}^{30}$.

\section{Future prospect of MS treatment}

Right now we have concentrated only on antiinflammatory process of MS. The good news is the ORATORIO study, which is with ocrelizumab, an anti-CD20 monoclonal antibody that was successful in PPMS about $24 \%$ reduction of the in terms of disability progression at least 12 weeks ${ }^{311}$.

It might be the strong anti-inflammatroy effect of the ocrelizumab or the $\mathrm{B}$ cell effect is different compare to fingolimod failed trial on PPMS.

In the future, we also need to apply for newer treatment strategy such as remyelination and/or neurodegeneration.

In remyelination process a possibility of antiLINGO-1 monoclonal antibody (BIIB033) therapy has some impact. RENEW clinical trial which was designed to detect whether treatment with antiLINGO-1 would result in remyelination. In this phase II clinical trial BIIB033 was compared to placebo in 82 people who'd recently had a first episode of optic neuritis (but did not have MS). Participants received a total of six intravenous infusions of the drug or placebo every four weeks and were followed up for a total of 32 weeks. BIIB033 was no better than placebo at improving vision, but researchers found that visual evoked potentials was improved $^{32)}$.

Autologous stem cell transplantation strategy for progressive MS failed but so far effective in the HALT-MS (high-dose immunosuppressive therapy and autologous hematopoietic cell transplantation for relapsing-remitting multiple sclerosis) study in aggressive relapsing-remitting MS at least three years. Improvement of EDSS scores from baseline suggested that nerves may be remyeli- nated. Off course this is theoretically be a one-time treatment that could lead to lifetime remission from MS, and have a chance for death by myeloablative treatment before transplantation and huge expense as a drawback ${ }^{33)}$.

Despite the numerous DMTs, long term effect and adverse effect is hard to estimate, especially in progressive MS. In the future, we need to supply tailor made therapy in each patient. For that purpose somatic cells from skin, blood or other tissues can be obtained from patients with different MS disease subtypes and reprogrammed to iPS cells will make accessible neural population as well as immune cells. The approach allows the establishment of subtype disease in a dish has the potential to identify novel pathogenetic mechanisms in MS as well as produce specific broad drug or toxicology screening during a drug development ${ }^{34}$.

\section{Adherence}

Adherence is another concern and MS special nurse have important role and unfortunately we have no MS special nurse in Japan even in our Juntendo hospital, therefore patients should be educated repeatedly after diagnosis and non-MS professional newrologists should know much about the risks and benefits of the newer DMTs.

During the session at outpatient clinic, clinicians spend more time, when assessed the patient clinical state and MRI imaging parameters of disease progression, and monitoring adverse effects, with these effort we could offer better care instead of cure of this intractable disease. Effective, safe and well-tolerated therapies and good relationship may improve adherence and better outcome.

\section{Concluding remarks}

As more effective DMTs become available for the treatment of MS in Japan compare to 10 years ago, second line DMTs such as injectable natalizumab and oral medicine fingolimod fulfill more patients' needs in terms of NEDA metrics in a short term. But long term efficacy of the progressive course and safety issues such as PML and malignancy are unknown. Fortunately, clinicians in Japan update information related DMTs easily, because of the drug lag at least 1 year, and 100 times of MS 
patients have already introduced different type of DMTs and we could learn safety profile and better treatment strategy in the real world setting. In the near future we hope to obtain new medicine for protecting the neurodegenerative process of progressive MS for cure.

\section{References}

1) Raza K, Filer A: The therapeutic window of opportunity in rheumatoid arthritis: does it ever close? Ann Rheum Dis, 2015; 74: 793-794.

2) Miller D, Barkhof F, Montalban X, Thompson A, Filippi M: Clinically isolated syndromes suggestive of multiple sclerosis, part I: natural history, pathogenesis, diagnosis, and prognosis. Lancet Neurol, 2005; 4: 281-288.

3) Tintore M: Rationale for early intervention with immunomodulatory treatments. J Neurol, 2008; 255 (Suppl 1): 37-43.

4) Arrambide G, Sastre-Garriga J: Predictive markers of disease evolution after a CIS in everyday practice. J Neurol Sci, 2014; 343: 8-14.

5) Havrdova E, Galetta S, Hutchinson M, et al: Effect of natalizumab on clinical and radiological disease activity in multiple sclerosis: a retrospective analysis of the Natalizumab Safety and Efficacy in Relapsing-Remitting Multiple Sclerosis (AFFIRM) study. Lancet Neurol, 2009; 8: 254-260.

6) West T: NEDA is central to current MS treatment. Neurology Reviews, 2015; 23: 14-17.

7) Rotstein DL, Healy BC, Malik MT, Chitnis T, Weiner HL: Evaluation of no evidence of disease activity in a 7-year longitudinal multiple sclerosis cohort. JAMA Neurol, 2015; 72: 152-158.

8) Cocco E, Sardu C, Spinicci G, et al: Influence of treatments in multiple sclerosis disability: a cohort study. Mult Scler, 2015; 21: 433-441.

9) Andersen O: Predicting a window of therapeutic opportunity in multiple sclerosis. Brain, 2010; 133 (Pt 7): 18631865.

10) Hansen K, Schüssel K, Kieble M, et al: Adherence to Disease Modifying Drugs among Patients with Multiple Sclerosis in Germany: A Retrospective Cohort Study. PLoS One, 2015; 10: e0133279.

11) Rio J, Castillo J, Rovira A, et al: Measures in the first year of therapy predict the response to interferon beta in MS. Mult Scler, 2009; 15: 848-853.

12) Sormani MP, Rio J, Tintore M, et al: Scoring treatment response in patients with relapsing multiple sclerosis. Mult Scler, 2013; 19: 605-612.

13) Rio J, Nos C, Tintore M, et al: Defining the response to interferon-beta in relapsing-remitting multiple sclerosis patients. Ann Neurol, 2006; 59: 344-352.
14) Nakatsuji Y, Okuno T, Moriya M, et al: Elevation of Sema4A implicates Th cell skewing and the efficacy of IFN- $\beta$ therapy in multiple sclerosis. J Immunol, 2012; 188: 4858-4865.

15) Sato DK, Nakashima I, Fukazawa T, et al: Neutralizing antibodies are associated with a reduction of interferon- $\beta$ efficacy during the treatment of Japanese multiple sclerosis patients. Tohoku J Exp Med, 2012; 228: 85-92.

16) Calabresi PA, Kieseier BC, Arnold DL, et al: Pegylated interferon $\beta$ - 1 a for relapsing-remitting multiple sclerosis (ADVANCE): a randomised, phase 3, double-blind study. Lancet Neurol, 2014; 13: 657-665.

17) La Mantia L, Di Pietrantonj C, Rovaris M, et al: Comparative efficacy of interferon $\beta$ versus glatiramer acetate for relapsing-remitting multiple sclerosis. J Neurol Neurosurg Psychiatry 2015; 86: 1016-1020.

18) Cohen JA, Barkhof F, Comi G, et al: Oral fingolimod or intramuscular interferon for relapsing multiple sclerosis. N Engl J Med, 2010; 362: 402-415.

19) Kappos L, Radue EW, O'Connor P, et al: A placebo-controlled trial of oral fingolimod in relapsing multiple sclerosis. N Engl J Med, 2010; 362: 387-401.

20) Ransohoff RM: Natalizumab for multiple sclerosis. N Engl J Med, 2007; 356: 2622-2629.

21) Langer-Gould A, Atlas SW, Green AJ, Bollen AW, Pelletier D: Progressive multifocal leukoencephalopathy in a patient treated with natalizumab. N Engl J Med, 2005; 353: 375-381.

22) Bloomgren G, Richman S, Hotermans C, et al: Risk of natalizumab-associated progressive multifocal leukoencephalopathy. N Engl J Med, 2012; 366: 1870-1880.

23) Calic Z, Cappelen-Smith C, Hodgkinson SJ, McDougall A, Cuganesan R, Brew BJ: Treatment of progressive multifocal leukoencephalopathy-immune reconstitution inflammatory syndrome with intravenous immunoglobulin in a patient with multiple sclerosis treated with fingolimod after discontinuation of natalizumab. J Clin Neurosci, 2015; 22: 598-600.

24) Calabresi PA, Giovannoni G, Confavreux C, et al; AFFIRM and SENTINEL Investigators: The incidence and significance of anti-natalizumab antibodies: results from AFFIRM and SENTINEL. Neurology, 2007; 69: 1391-1403.

25) Rigau V, Mania A, Béfort P, et al: Lethal multiple sclerosis relapse after natalizumab withdrawal. Neurology, 2012; 79: 2214-2216.

26) O' Connor PW, Goodman A, Kappos L, et al: Disease activity return during natalizumab treatment interruption in patients with multiple sclerosis. Neurology, 2011; 76: $1858-1865$.

27) Kappos L, Radue EW, Comi G; TOFINGO study group: Switching from natalizumab to fingolimod: A randomized, placebo-controlled study in RRMS. Neurology, 2015; 85: 29-39. 
28) Dong-Si T, Richman S, Wattjes MP, et al: Outcome and survival of asymptomatic PML in natalizumab-treated MS patients. Ann Clin Transl Neurol, 2014; 1: 755-764.

29) [No authors listed]BG 12: BG 00012, BG 12/Oral Fumarate, FAG-201, second-generation fumarate derivative --Fumapharm/Biogen Idec. Drugs R D, 2005; 6: 229230 .

30) Rosenkranz T, Novas M, Terborg C: PML in a patient with lymphocytopenia treated with dimethyl fumarate. N Engl J Med, 2015; 372: 1476-1478.

31) Montalban X, Hemmer B, Rammohan K, et al: Efficacy and safety of ocrelizumab in primary progressive multiple sclerosis: results of the phase III, double-blind, placebo-controlled ORATORIO study. Program and abstracts of the 31st Congress of the European Committee for Treatment and Research in Multiple Sclerosis
(ECTRIMS); October 7-10, 2015; Barcelona, Spain. Abstract 228.

32) Cadavid D, Balcer L, Galetta S, et al: Efficacy Analysis of the Anti-LINGO-1 Monoclonal Antibody BIIB033 in Acute Optic Neuritis: the RENEW Trial. The 67th annual meeting of the American Academy of Neurology took place in Washington, April 18-25.

33) Nash RA, Hutton GJ, Racke MK, et al: High-dose immunosuppressive therapy and autologous hematopoietic cell transplantation for relapsing-remitting multiple sclerosis (HALT-MS): a 3-year interim report. JAMA Neurol, 2015; 72: 159-169.

34) Di Ruscio A, Patti F, Welner RS, Tenen DG, Amabile G: Multiple sclerosis: getting personal with induced pluripotent stem cells. Cell Death Dis, 2015; 6: e1806. 